\title{
Public Good Provision and the Comparative Statics OF OPTIMAL NONLINEAR INCOME TAXATION
}

\author{
by
}

Craig Brett and John A. Weymark

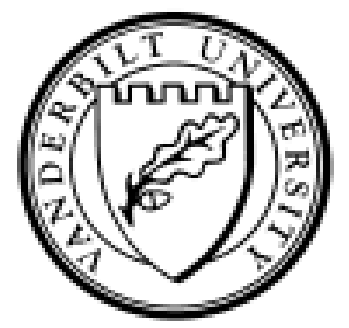

Working Paper No. 04-W15

June 2004

\section{DEPARTMENT OF ECONOMICS \\ VANDERBILT UNIVERSITY \\ NASHVILLE, TN 37235}

www.vanderbilt.edu/econ 


\title{
Public Good Provision and the Comparative Statics of Optimal Nonlinear Income Taxation*
}

\author{
by \\ Craig Brett \\ Department of Economics, \\ Mount Allison University, \\ 144 Main Street, Sackville NB, E4L 1A7, Canada \\ (e-mail: cbrett@mta.ca) \\ and \\ John A. Weymark \\ Department of Economics, \\ Box 1819, Station B, Vanderbilt University, \\ Nashville, TN 37235, U.S.A. \\ (e-mail: john.weymark@vanderbilt.edu)
}

June 2004

*Craig Brett's research is generously supported by the Canada Research Chair Programme and by the Canada Foundation for Innovation. 


\author{
Abstract \\ "Public Good Provision and the Comparative Statics \\ of Optimal Nonlinear Income Taxation" \\ by \\ Craig Brett and John A. Weymark
}

Comparative static properties of the solution to an optimal nonlinear income tax problem are provided for a model in which the government both designs an income tax schedule for redistributive purposes and provides a public good optimally. There are two types of individuals, distinguished by their skill levels, who have the same quasilinear preferences for labour supply and the consumption of a private and a public good. The parameters for which comparative statics are obtained are the weights in a weighted utilitarian social welfare function, the prices of the private and public goods, a taste parameter that measures the onerousness of working, and the individual skill levels.

Journal of Economic Literature classification numbers: D82, H21, H41.

Keywords and phrases: optimal income taxation, public goods, comparative statics. 


\section{Introduction}

There are relatively few comparative static results available for models of optimal nonlinear income taxation. Weymark (1987) has derived a number of comparative static properties of the solution to a finite population version of the Mirrlees (1971) nonlinear income tax problem for the case in which individual preferences are quasilinear. In the Mirrlees model, individuals only differ in their skill levels and the only role for the government is to design an income tax schedule for redistributive purposes. In this article, we extend Weymark's analysis by considering the implications for his results of adding public good provision as a second policy instrument for the government in an economy with two types of individuals. ${ }^{1}$

The main focus of the literature on optimal taxation with a public good has been the identification of conditions that characterize the optimal provision of the public good. In particular, considerable attention has been devoted to determining when the Samuelson (1954) Rule continues to hold in the absence of personalized lump-sum taxation, and to determining whether the Samuelson Rule leads to over- or under-provision when it is not optimal to employ this rule. Much of this analysis, which has its origins in the insightful discussion of Pigou (1947) on the use of distortionary taxes to finance public goods, has been conducted using models in which linear commodity taxes are the only available tax instruments. See Myles (1995) for an introduction to this literature.

Financing public good provision using nonlinear incomes taxes, either as a substitute for or as a supplement to commodity taxation, has been considered by Boadway and Keen (1993), Christiansen (1981), Edwards, Keen, and Tuomala (1994), Mirrlees (1976), Nava, Schroyen, and Marchand (1996), and Tuomala (1990), among others. When individuals have identical preferences and these preferences are represented by a strictly concave utility function, Christiansen (1981) and Boadway and Keen (1993) have shown that the Samuelson Rule for the optimal provision of a public good holds at the solution to the optimal nonlinear income tax problem if the utility function is weakly separable between consumption and labour supply. As a consequence, the public good serves no redistributive purpose when these assumptions are satisfied. We show that these conclusions also hold when preferences are quasilinear.

As in the Mirrlees model, we assume that individuals differ only in their labour productivities. Here, there are only two skill levels, as in Boadway and Keen (1993), Edwards, Keen, and Tuomala (1994), and Nava, Schroyen, and Marchand (1996). Individuals supply labour and consume a private and a public good. Everyone has the same

\footnotetext{
${ }^{1}$ Recently, a number of comparative static properties of optimal nonlinear income taxes when preferences are quasilinear have been developed for economies with only two possible skill levels. Hamilton and Pestieau (2002) have investigated the effects on the individuals' utilities of changing the skill distribution. Boadway and Pestieau (2004) have determined how the utility possibilities frontier responds to changes in the skill distribution and in the exogenous amount of revenue available for redistributive purposes. Cebreiro-Gómez (2002) has considered a two-country extension of the Weymark (1987) model and has identified the impact on the marginal tax rates faced by individuals of changing the producer prices in each of the countries.
} 
preferences and these preferences are represented by a quasilinear utility function that is linear in the amount of labour supplied. A linear production technology is used to produce the two consumption goods using labour in efficiency units as the sole input. The government, who knows the distribution of types, but does not know who has which skill level, chooses a nonlinear income tax schedule to maximize a weighted utilitarian social welfare function subject to a production-feasibility constraint and the constraint that each person's consumption bundle is optimal for him given the tax schedule. The parameters that are varied in our comparative static analysis are (i) the weights in the social welfare function, (ii) the prices (marginal costs) of the private and public goods, (iii) a taste parameter that measures the onerousness of working, and (iv) the skill levels of the two individuals. We are interested in determining how a marginal change in each of these parameters affects the optimal allocation of goods and services and the marginal tax rate faced by the low-skilled individual. ${ }^{2}$ Some clear-cut comparative statics for the optimal consumptions of the private and public goods are obtained for each of the parameter changes considered, but the results are less definitive for the other endogenous variables.

With our quasilinear preference assumption, the optimal consumptions of the private and public goods can be obtained by solving a "reduced-form" optimization problem that only involves these variables. The optimal incomes are functions of these optimal consumptions. In models without a public good, similar reduced-form problems were developed by Lollivier and Rochet (1983) for a continuum of individuals and by Weymark (1986) for a finite population. Weymark's reduced-form problem was used by Weymark (1987) to help obtain his comparative static results. A feature of the private good model with quasilinear preferences is that an explicit solution exists for the optimal consumptions as a function of the underlying parameters. This is not the case when there is also a public good. As a consequence, we have to employ different techniques to analyse the comparative static properties of our reduced-form problem than does Weymark (1987).

We present our model in Section 2. In Section 3, we establish some properties of the solution to our optimal nonlinear income tax problem and show how the optimal allocation can be obtained using a reduced-form optimization problem for the consumption variables. In Section 4, we use the first-order conditions from the reduced-form problem to obtain a matrix that contains expressions showing how to optimally change each of the consumption variables in response to a marginal increase in each of our parameters. Using this matrix, we develop our comparative static results for the technology and preference parameters in Section 5 and for the skill levels and welfare weights in Section 6 . Some possible extensions of our analysis are discussed in Section 7. Proofs of our results may be found in the Appendix.

\footnotetext{
${ }^{2}$ As is standard in models of this kind, it is always optimal for the high-skilled individual to face a zero marginal tax rate.
} 


\section{The Model}

There are two individuals, indexed by $i=1,2$. These individuals differ in ability, with an individual of type $i$ possessing skill level $w_{i}{ }^{3}$ It is assumed that $0<w_{1}<w_{2}$. As is customary in the nonlinear income tax literature, ability is interpreted as a measure of the effectiveness of an individual's labour input in producing goods. Individual $i$ 's labour supply in efficiency units is

$$
y_{i}=w_{i} l_{i}, \quad i=1,2,
$$

where $l_{i}$ is $i$ 's actual labour supply in, for example, hours.

Labour in efficiency units is a homogenous input that is used to produce a private good and a public good using a linear production technology. The input-output vector $\left(l_{1}, l_{2}, c, g\right) \in \mathbb{R}_{+}^{4}$ satisfies the economy's production constraint if

$$
p c+q g \leq \sum_{i=1}^{2} w_{i} l_{i},
$$

where $c$ and $g$ are, respectively, the quantities of the private and public goods produced. ${ }^{4}$ An allocation for this economy is a vector $\left(y_{1}, y_{2}, c_{1}, c_{2}, g\right) \in \mathbb{R}_{+}^{5}$, where $c_{i}$ is $i$ 's consumption of the private good. Because $\sum_{i=1}^{2} c_{i}=c$, it follows from (2.1) that (2.2) can be rewritten as

$$
p \sum_{i=1}^{2} c_{i}+q g \leq \sum_{i=1}^{2} y_{i} .
$$

An allocation satisfying (2.3) is production feasible.

The input and output markets are perfectly competitive. Thus, $p$ is the producer price of the private good, $q$ is the producer price of the public good, $w_{i}$ is person $i$ 's wage, and $y_{i}$ is $i$ 's before-tax income.

For each individual, a consumption bundle specifies this individual's labour supply and consumptions of the private and public goods. Both individuals have the same preferences for consumption bundles. These preferences are represented by the quasilinear utility function $u: \mathbb{R}_{+}^{3} \rightarrow \mathbb{R}$, where

$$
u\left(l_{i}, c_{i}, g\right)=v\left(c_{i}, g\right)-\gamma l_{i}
$$

The function $v$ is assumed to be twice continuously differentiable at all $\left(c_{i}, g\right) \neq(0,0)$, continuous and nondecreasing on $\mathbb{R}_{+}^{2}$, strictly increasing on $\mathbb{R}_{++}^{2}$, and strictly concave on $\mathbb{R}_{++}^{2}$ with $v(0,0)=0, v_{c_{i}}(0, g)=\infty$ for all $g>0, v_{g}\left(c_{i}, 0\right)=\infty$ for all $c_{i}>0$, $v_{c_{i}}\left(c_{i}, g\right) \rightarrow 0$ as $c_{i} \rightarrow \infty$ for all $g \geq 0$, and $v_{g}\left(c_{i}, g\right) \rightarrow 0$ as $g \rightarrow \infty$ for all $c_{i} \geq 0$. The

\footnotetext{
${ }^{3}$ All of the comparative static results presented here are also valid for an arbitrary number of individuals of each type.

${ }^{4} \mathbb{R}_{+}$and $\mathbb{R}_{++}$are, respectively, the nonnegative and positive subsets of the real line $\mathbb{R}$.
} 
limiting assumptions on $v$ ensure that the optimal tax problem has a solution and that both individuals have positive consumption of both goods in this solution. The taste parameter $\gamma$ is assumed to be positive. In the absence of the public good, preferences that are quasilinear in labour supply have been used by Boadway, Cuff, and Marchand (2000), Lollivier and Rochet (1983), Rochet (1987), and Weymark (1986, 1987), among others, to study optimal nonlinear income taxation. ${ }^{5}$

Some of our comparative static results depend on the sign of the cross partial derivative $v_{c_{i} g}\left(c_{i}, g\right)$. From Young's Theorem, we can interpret this derivative as either (i) the rate at which the marginal valuation of the public good changes in response to a marginal increase in the consumption of the private good or (ii) the rate at which the marginal utility of private consumption changes in response to a marginal increase in the quantity of the public good.

Using (2.1), the utility function in (2.4) can be rewritten in terms of the variables $y_{i}$, $c_{i}$, and $g$ that can be observed (and controlled) by the government. The resulting utility function $\tilde{U}^{i}: \mathbb{R}_{+}^{3} \rightarrow \mathbb{R}$ is person-specific and is given by

$$
\tilde{U}^{i}\left(y_{i}, c_{i}, g\right)=v\left(c_{i}, g\right)-\gamma y_{i} / w_{i}, \quad i=1,2 .
$$

As in Lollivier and Rochet (1983) and Weymark $(1986,1987)$, we work with the following transformation of the utility function:

$$
U^{i}\left(y_{i}, c_{i}, g\right)=w_{i} v\left(c_{i}, g\right)-\gamma y_{i}, \quad i=1,2 .
$$

The representation of preferences given in (2.6) is linear in the privately-observable parameter $w_{i}$. This feature of the utility function $U^{i}$ simplifies the analysis of the model. ${ }^{6}$

Person $i$ 's marginal rate of substitution between income and private consumption is

$$
\operatorname{MRS}_{y c}^{i}\left(y_{i}, c_{i}, g\right)=\gamma / w_{i} v_{c_{i}}\left(c_{i}, g\right), \quad i=1,2 .
$$

The quasilinearity assumption implies that this marginal rate of substitution is independent of income. The strict concavity of $v$ implies that $i$ 's marginal rate of substitution is increasing in private consumption. For a given value of $g$, the marginal rate of substitution is decreasing in $w_{i}$, so in terms of income-private consumption pairs, preferences satisfy the Mirrlees (1971)-Spence (1974) single-crossing property.

Person $i$ 's marginal rate of substitution between public and private consumption is

$$
\operatorname{MRS}_{g c}^{i}\left(y_{i}, c_{i}, g\right)=-\frac{v_{g}\left(c_{i}, g\right)}{v_{c_{i}}\left(c_{i}, g\right)}, \quad i=1,2 .
$$

As in (2.7), this marginal rate of substitution is independent of income.

\footnotetext{
${ }^{5}$ Diamond (1998) considers preferences that are quasilinear in consumption.

${ }^{6}$ In particular, this form of the utility function allows us to use techniques similar to those employed by Lollivier and Rochet (1983), Rochet (1987), and Weymark $(1986,1987)$.
} 
The taxation authority can observe the individual incomes and knows that one person has ability $w_{1}$ and one person has ability $w_{2}$, but does not know who is of which type. ${ }^{7}$ Furthermore, it cannot observe either person's labour supply. As a consequence, the government must raise the revenue it requires to pay for the public good and to pursue its redistributive goals using an anonymous tax schedule. Individuals choose their mostpreferred income-consumption bundles from this schedule. Thus, an allocation must satisfy the familiar incentive-compatibility (self-selection) conditions:

$$
U^{1}\left(y_{1}, c_{1}, g\right) \geq U^{1}\left(y_{2}, c_{2}, g\right)
$$

and

$$
U^{2}\left(y_{2}, c_{2}, g\right) \geq U^{2}\left(y_{1}, c_{1}, g\right) .
$$

Moreover, any allocation that satisfies (2.9) and (2.10) can be decentralized through the use of an anonymous taxation scheme. See, for example, Guesnerie and Seade (1983) or Guesnerie (1995). An allocation that satisfies (2.9) and (2.10) is incentive compatible.

A well-known consequence of incentive compatibility is that

$$
c_{1} \leq c_{2}
$$

and

$$
y_{1} \leq y_{2},
$$

with

$$
c_{1}=c_{2} \leftrightarrow y_{1}=y_{2} .
$$

If (2.13) holds, the two individuals are said to be bunched.

Individual tax liabilities are computed and collected on the basis of income, and any remaining personal income is spent on consumption of the private good. The value of private consumption by individual $i$ is $p c_{i}$, which is also $i$ 's after-tax income. The difference between aggregate before- and after-tax income is used to finance the provision of the public good. The government budget constraint is therefore given by

$$
q g \leq \sum_{i=1}^{2}\left(y_{i}-p c_{i}\right) .
$$

The equivalence of (2.3) and (2.14) follows from Walras' Law.

\footnotetext{
${ }^{7}$ In our model, we do not distinguish between the taxation authority and the government. In principle, the agencies that design the income tax schedule and that choose the level of the public good could be distinct.
} 
The government has preferences over allocations represented by a weighted utilitarian social welfare function. Using the transformed utilities in (2.6), the social welfare function is the function $\mathcal{W}: \mathbb{R}_{+}^{5} \rightarrow \mathbb{R}$ given by

$$
\mathcal{W}\left(y_{1}, y_{2}, c_{1}, c_{2}, g\right)=\lambda_{1} U^{1}\left(y_{1}, c_{1}, g\right)+\lambda_{2} U^{2}\left(y_{2}, c_{2}, g\right)
$$

It is assumed that the welfare weights, $\lambda_{1}$ and $\lambda_{2}$, are both positive with $\lambda_{1}>\lambda_{2}$. Because the underlying social welfare ordering only depends on the relative welfare weights, it is convenient to normalize these weights so that

$$
\lambda_{1}+\lambda_{2}=2 .
$$

With this normalization, changes in the relative welfare weights are fully described by changes in $\lambda_{1}$ alone. $^{8}$

Because the set of allocations that are incentive compatible is the same as the set of allocations that are achievable using anonymous tax schedules, the government's optimal nonlinear income tax problem can be described as follows.

The Optimal Nonlinear Income Tax Problem. The government chooses an allocation $\left(y_{1}, y_{2}, c_{1}, c_{2}, g\right) \in \mathbb{R}_{+}^{5}$ to maximize the social welfare function (2.15) subject to the production-feasibility constraint (2.3) and the incentive-compatibility constraints (2.9) and (2.10).

\section{Preliminary Results}

Weymark (1986) has analyzed a multi-person version of the model presented in the preceding section, but without the public good. He solved this problem in two steps. First, for fixed consumptions of the private good that are weakly monotonic in the ability parameter (as required by incentive compatibility), he determined the optimal incomes. By substituting the optimal values for the incomes into the objective function, the production-feasibility constraint, and the self-selection constraints, Weymark obtained a "reduced-form" optimization problem that he used to solve for the optimal consumptions. Although all incomes are nonnegative at the solution to his optimal nonlinear income taxation problem, in order to avoid some technical complications that arise when the lowest type does not work, in the first step of his procedure, Weymark permitted some incomes to be negative for non-optimal values of the consumptions. As in Weymark (1986), we develop a reduced-form optimization problem for our model with a public good to solve for the optimal consumptions. However, in contrast to his analysis, we only determine the optimal incomes corresponding to the optimal consumptions. We

\footnotetext{
${ }^{8}$ Note that the sum of the welfare weights is equal to the number of consumers. It is also possible to define the social welfare function using the untransformed utility functions $\tilde{U}^{i}$ in (2.4). An equal-weight utilitarian social welfare function defined using these utility functions places higher weight on person one when rewritten in terms of the transformed utility functions. See Weymark (1986) for details.
} 
assume that the underlying parameters of our problem are such that it is optimal for everyone to have a positive income, thereby avoiding the boundary problems mentioned above.

We begin by determining which of the constraints bind at a solution to the optimal nonlinear income tax problem. As is standard in nonlinear income tax problems with a single budget constaint, it is optimal for the production constraint to bind. ${ }^{9}$ When there are only two types of individuals, at most one of the self-selection constraints binds. Which, if any, of these constraints bind depends on the relative values of the welfare weights. ${ }^{10}$ Our assumption that $\lambda_{1}>\lambda_{2}$ implies that the optimal tax problem is redistributive, in the sense of Guesnerie (1995). That is, in the neighbourhood of the optimal allocation, a redistribution of private consumption from the high-productivity individual to the low-productivity individual and/or a redistribution of before-tax income from the low-productivity individual to the high-productivity individual is socially desirable, but would violate the incentive-compatibility constraints. As a consequence, it is optimal for person two's, but not person one's, incentive-compatibility constraint to bind at the solution to the optimal tax problem. Because bunching can only occur if both incentive constraints bind, the individuals are not bunched.

Lemma 1. If $\left(\tilde{y}_{1}, \tilde{y}_{2}, \tilde{c}_{1}, \tilde{c}_{2}, \tilde{g}\right)$ is a solution to the optimal nonlinear income tax problem with $\tilde{y}_{1}>0$, then

$$
\begin{gathered}
p\left(\tilde{c}_{1}+\tilde{c}_{2}\right)+q \tilde{g}=\tilde{y}_{1}+\tilde{y}_{2}, \\
w_{2} v\left(\tilde{c}_{2}, \tilde{g}\right)-\gamma \tilde{y}_{2}=w_{2} v\left(\tilde{c}_{1}, \tilde{g}\right)-\gamma \tilde{y}_{1},
\end{gathered}
$$

and

$$
w_{1} v\left(\tilde{c}_{1}, \tilde{g}\right)-\gamma \tilde{y}_{1}>w_{1} v\left(\tilde{c}_{2}, \tilde{g}\right)-\gamma \tilde{y}_{2}
$$

Furthermore, the individuals are not bunched in this allocation. ${ }^{11}$

The optimal nonlinear income taxation problem can be solved in two steps. First, the optimal values for the consumptions of the private and public goods are determined. Then, using these values, the optimal incomes are obtained by simultaneously solving (3.1) and (3.2).

The reduced-form objective function in the first step is the function $W: \mathbb{R}_{+}^{3} \rightarrow \mathbb{R}$ given by

$$
W\left(c_{1}, c_{2}, g\right)=\beta_{1} v\left(c_{1}, g\right)+\beta_{2} v\left(c_{2}, g\right)-\gamma p\left(c_{1}+c_{2}\right)-\gamma q g,
$$

\footnotetext{
${ }^{9}$ Production inefficiency may be optimal when there are multiple budgets constraints. See, for example, Cebreiro-Gómez (2002).

${ }^{10}$ See Stiglitz (1983) for a detailed discussion of this issue.

${ }^{11}$ The standard proofs of Lemma 1 apply to models in which utility is not linear in before-tax income.
} 
where

$$
\beta_{1}=w_{1}+\left(1-\lambda_{1}\right)\left(w_{2}-w_{1}\right)
$$

and

$$
\beta_{2}=w_{2}
$$

The normalization $\lambda_{1}+\lambda_{2}=2$ and the assumptions that $\lambda_{1}>\lambda_{2}$ and $w_{2}>w_{1}$ imply that $\beta_{2}>w_{1}>\beta_{1}$.

The description of the first-stage reduced-form optimization problem is completed by specifying the constraints on $c_{1}, c_{2}$, and $g$.

The Reduced-Form Optimization Problem. The government chooses $\left(c_{1}, c_{2}, g\right) \in$ $\mathbb{R}_{+}^{3}$ to maximize $W\left(c_{1}, c_{2}, g\right)$ subject to the monotonicity constraint $(2.11)$ on the private consumptions.

Lemma 2 shows that the solution to the reduced-form optimization problem is unique and that it is an unconstrained maximum of the reduced-form objective function $W$.

Lemma 2. There is a unique solution $\left(\tilde{c}_{1}, \tilde{c}_{2}, \tilde{g}\right)$ to the reduced-form optimization problem. In this solution, $\tilde{c}_{2}>\tilde{c}_{1}>0$ and $\tilde{g}>0 .{ }^{12}$

When the production-feasibility constraint and person two's self-selection constraint both bind, the value of the social welfare function $\mathcal{W}$ at the allocation $\left(y_{1}, y_{2}, c_{1}, c_{2}, g\right)$ is the same as the value of the reduced-form objective function $W$ at $\left(c_{1}, c_{2}, g\right)$.

Lemma 3. If $\left(y_{1}, y_{2}, c_{1}, c_{2}, g\right)$ satisfies (3.1) and (3.2), then

$$
\mathcal{W}\left(y_{1}, y_{2}, c_{1}, c_{2}, g\right)=W\left(c_{1}, c_{2}, g\right)
$$

Proposition 1 shows that the optimal consumptions of the private and public goods are obtained by solving the reduced-form optimization problem, with the optimal incomes given by (3.8) and (3.9), provided that person one's optimal income is positive.

Proposition 1. The allocation $\left(\tilde{y}_{1}, \tilde{y}_{2}, \tilde{c}_{1}, \tilde{c}_{2}, \tilde{g}\right)$ is (i) a solution to the optimal nonlinear income tax problem with $\tilde{y}_{1}>0$ if and only if (ii) $\left(\tilde{c}_{1}, \tilde{c}_{2}, \tilde{g}\right)$ solves the reduced-form optimization problem,

$$
\tilde{y}_{1}=\frac{1}{2}\left\{p \sum_{i=1}^{2} \tilde{c}_{i}+q \tilde{g}-\frac{1}{\gamma} w_{2}\left[v\left(\tilde{c}_{2}, \tilde{g}\right)-v\left(\tilde{c}_{1}, \tilde{g}\right)\right]\right\}>0,
$$

\footnotetext{
${ }^{12}$ Because the solution to the reduced-form problem is strictly positive, in our comparative static analysis, we simply say that $v$ is strictly increasing and strictly concave without adding the proviso that these properties of $v$ only hold on $\mathbb{R}_{++}^{2}$.
} 
and

$$
\tilde{y}_{2}=\frac{1}{2}\left\{p \sum_{i=1}^{2} \tilde{c}_{i}+q \tilde{g}+\frac{1}{\gamma} w_{2}\left[v\left(\tilde{c}_{2}, \tilde{g}\right)-v\left(\tilde{c}_{1}, \tilde{g}\right)\right]\right\} .
$$

It follows from Proposition 1 and the fact that the reduced-form optimization problem has a unique solution that there is also a unique solution to the optimal nonlinear income tax problem.

The preceding results can be used to help interpret the weights $\beta_{1}$ and $\beta_{2}$ that appear in the reduced-form objective function. The wage $w_{i}$ measures the social value of an additional unit of utility for person $i$ obtained from the consumption of either the private or the public good. In contrast, the weight $\beta_{i}$ measures the social value of an additional unit of utility for person $i$ from the consumption of these goods, adjusted for the additional costs that this utility might impose through the tightening of an incentivecompatibility constraint. Because person two's incentive-compatibility constraint binds, the direct gains to social welfare from an increase in person one's private consumption are partly offset by the losses due to the tightening of the binding incentive constraint. For this reason, $\beta_{1}<w_{1}$. On the other hand, because person one's incentive-compatibility constraint does not bind, person two's private consumption can be increased without violating an incentive constraint, and so no adjustment to the social value of two's utility is needed; i.e., $\beta_{2}=w_{2}$.

Because none of the constraints bind, the first-order conditions for a solution to the reduced-form optimization problem are given by

$$
\frac{\partial W\left(c_{1}, c_{2}, g\right)}{\partial c_{i}}=\beta_{i} \frac{\partial v\left(c_{i}, g\right)}{\partial c_{i}}-\gamma p=0, \quad i=1,2
$$

and

$$
\frac{\partial W\left(c_{1}, c_{2}, g\right)}{\partial g}=\beta_{1} \frac{\partial v\left(c_{1}, g\right)}{\partial g}+\beta_{2} \frac{\partial v\left(c_{2}, g\right)}{\partial g}-\gamma q=0
$$

These first-order conditions have simple interpretations. For each person's consumption of the private good and for the public good, social marginal benefit, suitably adjusted for incentive effects, is equated with social marginal cost. In the case of the public good, the social marginal benefit is obtained by adding each individual's contribution to the social benefit, whereas with the private good, their social benefits are considered separately. Social marginal cost is measured in terms of utility. Hence, the social marginal cost of a unit of the private (resp. public) good is equal to the value of the labour used to produce that unit, $p$ (resp. $q$ ), multiplied by the marginal disutility of labour in efficiency units, $\gamma$.

The qualitative features of the optimal values for the marginal rates of income tax and the rule governing the optimal provision of the public good can be obtained using 
the first-order optimality conditions (3.10) and (3.11). Optimal income tax schedules are non-differentiable when there are a finite number of individuals, so the marginal tax rate faced by an individual is best defined by considering a personalized, differentiable tax schedule that results in the same choice of labour supply and private consumption as is obtained with the actual anonymous tax schedule. If person $i$ 's tax schedule were smooth at his optimal choice, at the margin, for each additional dollar of before-tax income, he would obtain an additional $\operatorname{MRS}_{y c}^{i}\left(y_{i}, c_{i}, g\right)$ units of the private good. The value of this additional consumption is $p \operatorname{MRS}_{y c}^{i}\left(y_{i}, c_{i}, g\right)$. Hence, using (2.7), person $i$ 's effective marginal tax rate is given by

$$
\operatorname{MTR}^{i}\left(y_{i}, c_{i}, g\right)=1-\gamma p / w_{i} v_{c_{i}}\left(c_{i}, g\right), \quad i=1,2 .
$$

Because preferences are quasilinear, this tax rate only depends on $i$ 's consumption of the private and public goods.

It is well-known that when preferences are strictly quasiconcave, it is optimal in a two-type economy for the low-productivity individual to face a marginal tax rate strictly between zero and one and it is optimal for the high-productivity individual to face a zero marginal tax rate. Further, as shown by Christiansen (1981, Proposition 1) and Boadway and Keen (1993, Corollary 1), when preferences are weakly separable between the consumption of the private and public goods and labour supply, the Samuelson (1954) Rule for the optimal provision of the public good holds (the sum of the marginal rates of substitution between public and private consumption should equal the marginal rate of transformation between these two goods). ${ }^{13}$ These properties of the solution to the optimal income tax problem are confirmed in Proposition 2 for the economy with quasilinear preferences considered here.

Proposition 2. If $\left(\tilde{y}_{1}, \tilde{y}_{2}, \tilde{c}_{1}, \tilde{c}_{2}, \tilde{g}\right)$ is a solution to the optimal nonlinear income tax problem with $\tilde{y}_{1}>0$, then

$$
\begin{gathered}
0<\operatorname{MTR}^{1}\left(\tilde{y}_{1}, \tilde{c}_{1}, \tilde{g}\right)<1, \\
\operatorname{MTR}^{2}\left(\tilde{y}_{2}, \tilde{c}_{2}, \tilde{g}\right)=0,
\end{gathered}
$$

and

$$
\operatorname{MRS}_{g c}^{1}\left(\tilde{y}_{1}, \tilde{c}_{1}, \tilde{g}\right)+\operatorname{MRS}_{g c}^{2}\left(\tilde{y}_{2}, \tilde{c}_{2}, \tilde{g}\right)=-\frac{q}{p}
$$

\footnotetext{
${ }^{13}$ In the absence of the separability assumption, an additional term is added to the right-hand side of (3.15) that takes account of the difference between the two individuals' marginal rates of substitution at person one's commodity bundle. We follow Christiansen (1981) and Boadway and Keen (1993) by using private consumption as the numeraire good. The form of the optimality condition for the public good is different if, as in Mirrlees (1976) and Tuomala (1990), before-tax income is used as the numeraire; i.e., if the marginal rates of substitution in the optimality condition are between public good consumption and before-tax income. See Boadway and Keen (1993) for a detailed discussion of the numeraire issue.
} 


\section{A Comparative Static Matrix}

The optimal allocation, and hence the choice of an optimal tax schedule, depends on the underlying parameters of the economy. In our model, these exogenous variables are (i) the preference parameter $\gamma$, which measures the onerousness of labour effort, (ii) the producer prices $p$ and $q$, which characterize the production technology, (iii) the skill levels $w_{1}$ and $w_{2}$ of the two individuals, and (iv) the weights $\lambda_{1}$ and $\lambda_{2}$ in the social welfare function. We are interested in determining the qualitative properties of how the optimal allocation varies in response to marginal changes in these parameters.

Weymark (1987) analyzed the comparative static properties of the optimal allocation for a multi-person version of our model in which there is no public good. When there is no public good, the analogue to the first-order condition (3.10) can be solved explicitly for person $i$ 's optimal consumption. Having explicit solutions for the optimal consumptions greatly facilitates the analysis of the comparative static properties of Weymark's model.

Without a specific functional form for $v$, it is not possible to explicitly solve the firstorder conditions (3.10) and (3.11) for the optimal allocation of the private and public goods. Nevertheless, the simplicity of the first-order conditions allows for a tractable analysis of the effects of changes in the parameters of our model of the economy on these variables. In the reduced-form optimization problem, the skill levels $w_{1}$ and $w_{2}$ and the welfare weights $\lambda_{1}$ and $\lambda_{2}$ only affect the optimal choice of the private and public consumptions through their effect on the adjusted welfare weights $\beta_{1}$ and $\beta_{2}$. In this section, we derive formulae that describe how to optimally change the three consumption variables in response to a marginal increase in $p, q, \gamma, \beta_{1}$, and $\beta_{2}$. These formulae are used in subsequent sections to determine the qualitative comparative static properties of our model.

For any positive values of the parameters $q, p, \gamma, \beta_{1}$, and $\beta_{2}$, we know from Lemma 2 that the reduced-form problem has a unique solution and that the optimal values for the three consumption variables are positive. Let $F: \mathbb{R}_{++}^{5} \rightarrow \mathbb{R}_{++}^{3}$ be the function mapping $\left(q, p, \gamma, \beta_{1}, \beta_{2}\right)$ to the optimal choice of $\left(c_{1}, c_{2}, g\right)$. Let $c_{1}^{*}: \mathbb{R}_{++}^{5} \rightarrow \mathbb{R}_{++}, c_{2}^{*}: \mathbb{R}_{++}^{5} \rightarrow$ $\mathbb{R}_{++}$, and $g^{*}: \mathbb{R}_{++}^{5} \rightarrow \mathbb{R}_{++}$denote the three component functions of $F$. Thus, for all $\left(q, p, \gamma, \beta_{1}, \beta_{2}\right) \in \mathbb{R}_{++}^{5}$,

$$
F\left(q, p, \gamma, \beta_{1}, \beta_{2}\right)=\left[c_{1}^{*}\left(q, p, \gamma, \beta_{1}, \beta_{2}\right), c_{2}^{*}\left(q, p, \gamma, \beta_{1}, \beta_{2}\right), g^{*}\left(q, p, \gamma, \beta_{1}, \beta_{2}\right)\right] .
$$

In Proposition 3, we show that $F$ is continuously differentiable and provide formulae for the partial derivatives of $F$.

Proposition 3. The solution function $F: \mathbb{R}_{++}^{5} \rightarrow \mathbb{R}_{++}^{3}$ of the reduced-form optimization problem is continuously differentiable. For all $\left(q, p, \gamma, \beta_{1}, \beta_{2}\right) \in \mathbb{R}_{++}^{5}$, the derivative DF of $F$ at $\left(q, p, \gamma, \beta_{1}, \beta_{2}\right)$ is given by

$$
D F\left(q, p, \gamma, \beta_{1}, \beta_{2}\right)=\left(A^{-1} B\right)\left(q, p, \gamma, \beta_{1}, \beta_{2}\right),
$$


where

$$
A\left(q, p, \gamma, \beta_{1}, \beta_{2}\right)=\left[\begin{array}{ccc}
\beta_{1} v_{c_{1} c_{1}}\left(c_{1}, g\right) & 0 & \beta_{1} v_{c_{1} g}\left(c_{1}, g\right) \\
0 & \beta_{2} v_{c_{2} c_{2}}\left(c_{2}, g\right) & \beta_{2} v_{c_{2} g}\left(c_{2}, g\right) \\
\beta_{1} v_{c_{1} g}\left(c_{1}, g\right) & \beta_{2} v_{c_{2} g}\left(c_{2}, g\right) & \beta_{1} v_{g g}\left(c_{1}, g\right)+\beta_{2} v_{g g}\left(c_{2}, g\right)
\end{array}\right]
$$

and

$$
B\left(q, p, \gamma, \beta_{1}, \beta_{2}\right)=\left[\begin{array}{ccccc}
0 & \gamma & p & -v_{c_{1}}\left(c_{1}, g\right) & 0 \\
0 & \gamma & p & 0 & -v_{c_{2}}\left(c_{2}, g\right) \\
\gamma & 0 & q & -v_{g}\left(c_{1}, g\right) & -v_{g}\left(c_{2}, g\right)
\end{array}\right]
$$

Henceforth, the dependence of matrices and functions on the values of the arguments at which they are evaluated is suppressed in our notation. When necessary to avoid abiguities by doing so, we index derivatives with respect to $g$ by the individual concerned. Thus,

$$
v_{g_{i}}=v_{g}\left(c_{i}, g\right) \quad \text { and } \quad v_{g_{i} g_{i}}=v_{g g}\left(c_{i}, g\right), \quad i=1,2 \text {. }
$$

We also let

$$
\mu=\beta_{1} v_{g g}\left(c_{1}, g\right)+\beta_{2} v_{g g}\left(c_{2}, g\right) .
$$

The entries in the matrix $D F$ in Proposition 3 are the partial derivatives of the solution functions $c_{1}^{*}, c_{2}^{*}$, and $g^{*}$ of the reduced-form optimization problem with respect to the five parameters. Our objective is to sign these partial derivatives. In order to do so, we need to find an explicit solution to (4.2).

Using the well-known formula for the inverse of a $3 \times 3$ matrix, we obtain

$$
A^{-1}=\frac{1}{|A|}\left[\begin{array}{ccc}
\beta_{2} v_{c_{2} c_{2}} \mu-\left(\beta_{2} v_{c_{2} g}\right)^{2} & \beta_{1} \beta_{2} v_{c_{1} g} v_{c_{2} g} & -\beta_{1} \beta_{2} v_{c_{2} c_{2}} v_{c_{1} g} \\
\beta_{1} \beta_{2} v_{c_{1} g} v_{c_{2} g} & \beta_{1} v_{c_{1} c_{1}} \mu-\left(\beta_{1} v_{c_{1} g}\right)^{2} & -\beta_{1} \beta_{2} v_{c_{1} c_{1}} v_{c_{2} g} \\
-\beta_{1} \beta_{2} v_{c_{2} c_{2}} v_{c_{1} g} & -\beta_{1} \beta_{2} v_{c_{1} c_{1}} v_{c_{2} g} & \beta_{1} \beta_{2} v_{c_{1} c_{1}} v_{c_{2} c_{2}}
\end{array}\right] .
$$

Using (4.7), the matrix $A^{-1} B$ can now be computed. Because $A^{-1} B$ is quite large, we present it column-by-column, with the following partition:

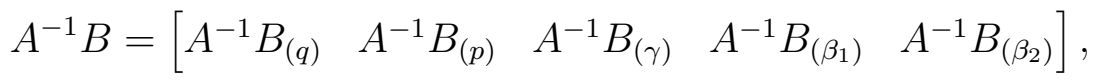

where

$$
\begin{gathered}
A^{-1} B_{(q)}=\frac{1}{|A|}\left[\begin{array}{c}
-\gamma \beta_{1} \beta_{2} v_{c_{2} c_{2}} v_{c_{1} g} \\
-\gamma \beta_{1} \beta_{2} v_{c_{1} c_{1}} v_{c_{2} g} \\
\gamma \beta_{1} \beta_{2} v_{c_{1} c_{1}} v_{c_{2} c_{2}}
\end{array}\right], \\
A^{-1} B_{(p)}=\frac{1}{|A|}\left[\begin{array}{c}
\gamma\left\{\beta_{2} v_{c_{2} c_{2}} \mu-\left(\beta_{2} v_{c_{2} g}\right)^{2}+\beta_{1} \beta_{2} v_{c_{1} g} v_{c_{2} g}\right\} \\
\gamma\left\{\beta_{1} v_{c_{1} c_{1}} \mu-\left(\beta_{1} v_{c_{1} g}\right)^{2}+\beta_{1} \beta_{2} v_{c_{1} g} v_{c_{2} g}\right\} \\
-\gamma\left\{\beta_{1} \beta_{2} v_{c_{1} c_{1}} v_{c_{2} g}+\beta_{1} \beta_{2} v_{c_{2} c_{2}} v_{c_{1} g}\right\}
\end{array}\right],
\end{gathered}
$$




$$
\begin{gathered}
A^{-1} B_{(\gamma)}=\frac{1}{|A|}\left[\begin{array}{c}
p\left\{\beta_{2} v_{c_{2} c_{2}} \mu-\left(\beta_{2} v_{c_{2} g}\right)^{2}+\beta_{1} \beta_{2} v_{c_{1} g} v_{c_{2} g}\right\}-q \beta_{1} \beta_{2} v_{c_{2} c_{2}} v_{c_{1} g} \\
p\left\{\beta_{1} v_{c_{1} c_{1}} \mu-\left(\beta_{1} v_{c_{1} g}\right)^{2}+\beta_{1} \beta_{2} v_{c_{1} g} v_{c_{2} g}\right\}-q \beta_{1} \beta_{2} v_{c_{1} c_{1}} v_{c_{2} g} \\
-p\left\{\beta_{1} \beta_{2} v_{c_{2} c_{2}} v_{c_{1} g}+\beta_{1} \beta_{2} c_{c_{1} c_{1}} v_{c_{2} g}\right\}+q \beta_{1} \beta_{2} v_{c_{1} c_{1}} v_{c_{2} c_{2}}
\end{array}\right], \\
A^{-1} B_{\left(\beta_{1}\right)}=\frac{1}{|A|}\left[\begin{array}{c}
-v_{c_{1}}\left\{\beta_{2} v_{c_{2} c_{2}} \mu-\left(\beta_{2} v_{c_{2} g}\right)^{2}\right\}+v_{g_{1}} \beta_{1} \beta_{2} v_{c_{2} c_{2}} v_{c_{1} g} \\
-v_{c_{1}} \beta_{1} \beta_{2} v_{c_{1} g} v_{c_{2} g}+v_{g_{1}} \beta_{1} \beta_{2} v_{c_{1} c_{1}} v_{c_{2} g} \\
v_{c_{1}} \beta_{1} \beta_{2} v_{c_{2} c_{2}} v_{c_{1} g}-v_{g_{1}} \beta_{1} \beta_{2} v_{c_{1} c_{1}} v_{c_{2} c_{2}}
\end{array}\right],
\end{gathered}
$$

and

$$
A^{-1} B_{\left(\beta_{2}\right)}=\frac{1}{|A|}\left[\begin{array}{c}
-v_{c_{2}} \beta_{1} \beta_{2} v_{c_{1} g} v_{c_{2} g}+v_{g_{2}} \beta_{1} \beta_{2} v_{c_{2} c_{2}} v_{c_{1} g} \\
-v_{c_{2}}\left\{\beta_{1} v_{c_{1} c_{1}} \mu-\left(\beta_{1} v_{c_{1} g}\right)^{2}\right\}+v_{g_{2}} \beta_{1} \beta_{2} v_{c_{1} c_{1}} v_{c_{2} g} g \\
v_{c_{2}} \beta_{1} \beta_{2} v_{c_{1} c_{1}} v_{c_{2} g}-v_{g_{2}} \beta_{1} \beta_{2} v_{c_{1} c_{1}} v_{c_{2} c_{2}}
\end{array}\right] .
$$

Each column in the partition of $A^{-1} B$ in (4.8) contains the comparative static information with respect to the parameter for which the column is labelled. The rows of $A^{-1} B$ contain the comparative static information for $c_{1}^{*}, c_{2}^{*}$, and $g^{*}$, respectively. In the next two sections, we investigate the signs of the entries in the comparative static matrix $A^{-1} B$. Note that each of these entries contains a common factor, $1 /|A|$. In the course of proving Proposition 3, we have shown that $|A|$ is negative.

\section{Comparative Statics for the Technology and Preference Pa- rameters}

Henceforth, let $\left(\bar{q}, \bar{p}, \bar{\gamma}, \bar{\lambda}_{1}, \bar{\lambda}_{2}, \bar{w}_{1}, \bar{w}_{2}\right) \in \mathbb{R}_{++}^{7}$ denote the initial values of the parameters that appear in our model and let $\bar{a}=\left(\bar{y}_{1}, \bar{y}_{2}, \bar{c}_{1}, \bar{c}_{2}, \bar{g}\right)$ denote the corresponding optimal allocation. We suppose that $\bar{y}_{1}$ (and, hence, every component of $\bar{a}$ ) is positive. For each of the parameters, our objective is to determine the direction of change in each of the components of the optimal allocation and in person one's marginal tax rate induced by a marginal increase in the value of the parameter. ${ }^{14}$ Our strategy is to use the comparative static matrix $A^{-1} B$ from Section 4 to sign the directions of change in the optimal consumptions of the private and public goods, (3.8) and (3.9) to sign the directions of change in the optimal before-tax incomes, and (3.12) to sign the direction of change in person one's marginal tax rate. ${ }^{15}$ By making assumptions about how the marginal valuation of the public good varies in response to a change in the consumption of the private good, for each of our parameters, we are able to obtain comparative static results for the consumption variables. Unfortunately, our model yields only a few unambiguous comparative statics for the income variables and for person one's marginal tax rate.

In this section, we investigate the impact of marginal changes in the technology parameters $p$ and $q$ and the taste parameter $\gamma$ on the solution to the optimal nonlinear

\footnotetext{
${ }^{14}$ Recall from Proposition 2 that it is always optimal for person two to face a zero marginal tax rate.

${ }^{15}$ Note that if an individual's skill level is not the parameter being changed, his before-tax income increases if and only if his labour supply increases.
} 
income tax problem and on person one's marginal tax rate. Because the welfare weights and skill levels are being held fixed, it follows from (3.5) and (3.6) that the initial values of the adjusted welfare weights, $\bar{\beta}_{1}$ and $\bar{\beta}_{2}$, are also being held constant.

\subsection{Changing the Price of the Public Good}

The considerable growth of the public sector in Western democracies from the end of the Second World War until the 1980s led some commentators to argue that the cost of government services increased relative to the cost of private sector activities over this period. In Canada, for example, the price index for government consumption increased faster than the overall price index from 1961 to $1990 .{ }^{16}$ In our model, the implications of such a trend on optimal tax policy can be captured by considering the impact that a change in the producer price $q$ of the public good has on the optimal allocation. Given the separability of preferences, the provision of the public good has no direct redistributive effects, so our comparative static results for changes in $q$ can be interpreted more broadly as identifying the effects of an increase in the cost of non-redistributive programmes on the structure of the government's redistributive and non-redistributive programmes.

Proposition 4 presents our comparative static results for a change in the price of the public good.

Proposition 4. If person one's income is positive in the initial optimal allocation, then a marginal increase in the price $q$ of the public good results in

(i) a decrease in the optimal provision of the public good,

(ii) an increase in person i's optimal consumption of the private good if and only if $i$ 's marginal utility of private consumption is decreasing in the consumption of the public good at the initial allocation, $i=1,2$, and

(iii) no change in the marginal income tax rate faced by person one.

The conclusion in part (i) of Proposition 4 is as one would expect. If the public good becomes more expensive, it is optimal to provide less of it.

Because of the separability in the reduced-form objective function, part (ii) of Proposition 4 can be interpreted using a first-best style of reasoning. For concreteness, consider the case in which the marginal utility of $i$ 's private consumption decreases with an increase in the provision of the public good. Recall from the discussion of (3.10) that at $\left(\bar{c}_{i}, \bar{g}\right)$, the social marginal benefit of an increase in person $i$ 's private consumption is $\bar{\beta}_{i} v_{c_{i}}$ and the social marginal cost is $\bar{\gamma} \bar{p}$. Because the values of $\beta_{1}, \gamma$, and $p$ are unaffected by a change in the price of the public good, if $i$ 's consumption of the private good is held fixed when the provision of the public good falls in response to an increase in its price,

\footnotetext{
${ }^{16}$ According to Statistics Canada (1998, Table 26, pp. 199-217), the ratio of the implicit price index of current government expenditure to the overall GDP deflator rose from 0.69 to 0.98 during this period.
} 
then the social marginal benefit of $i$ 's private consumption exceeds it social marginal cost. Therefore, it is optimal to increase $i$ 's consumption of this good.

The conclusion in part (iii) of Proposition 4 that person one's marginal tax rate (not just the marginal tax rate of person two) is unaffected by a change in the price of the public good is quite striking. The reasoning used to establish this result applies to both individuals. An implication of (3.10), the requirement the social marginal benefit of an individual's private consumption should equal its social marginal cost, is that

$$
\frac{\partial v\left(c_{i}^{*}\left(q, p, \gamma, \beta_{1}, \beta_{2}\right), g^{*}\left(q, p, \gamma, \beta_{1}, \beta_{2}\right)\right)}{\partial c_{i}}=\frac{\gamma p}{\beta_{i}}, \quad i=1,2
$$

Because the right-hand side of (5.1) does not depend on $q$, neither does its left-hand side. As a consequence, adjustments made to an individual's consumption of the private and public goods owing to a change in the price of the public good exactly offset each other in their effects on the marginal utility of his private consumption. Hence, from (3.12), there is no change in the marginal tax rate.

Except in special cases, it is not possible to sign the effects on the optimal incomes of an increase in the price of the public good. Suppose that a marginal change in $g$ has no affect on either individual's marginal utility of private consumption at the initial allocation. We know that in this case, it is optimal to decrease public good provision when $q$ increases, but not to change private consumption. If the marginal utility of private consumption does not change at any level of consumption (not just at $\bar{c}_{1}$ and $\bar{c}_{2}$ ) when we decrease the public good level to its new optimal level, then by (2.7), the indifference curves in income-private consumption space remain the same. As a consequence, person two's incentive-compability constraint still binds when $g$ is decreased holding the other components of the allocation fixed. Thus, only the middle term in the equations for the optimal incomes, (3.8) and (3.9), is affected by the increase in $q$. Because preferences are quasilinear, to satisfy the production-feasiblity constraint with equality, the incomes of both individuals need to be increased (resp. decreased) by a common amount if the total cost $q g$ of the public good has increased (resp. decreased).

If, however, the marginal utility of private consumption does change for some consumption levels different from $\bar{c}_{1}$ and $\bar{c}_{2}$ (but not at $\bar{c}_{1}$ and $\bar{c}_{2}$ ), then the indifference curves in income-private consumption space change when $g$ is decreased, and two's incentivecompatability constraint need not bind (nor even be satisfied). Thus, in this case, the value of the third term in (3.8) and (3.9) may not be invariant to a change in $g$. Holding $y_{1}$ at its initial value, $y_{2}$ must be increased (resp. decreased) to restore the satisfaction of two's incentive constraint with equality if the last term in (3.9) has increased (resp. decreased) before implementing a common change in the two incomes in order to satisfy the production-feasibility constraint with equality.

If a change in $g$ does affect either individual's marginal utility of private consumption at the initial allocation, then it becomes necessary to take account of all of the terms in (3.8) and (3.9) when determining how the optimal incomes change. That is, when $g$ decreases, we need to know what happens to the total cost of both private and public 
consumption and we need to know which responds more to the change in $g, v_{c_{1}}$ or $v_{c_{2}}$. There is no reason, in general, for these effects to reinforce one another.

\subsection{Changing the Price of the Private Good}

In a version of our model without public good provision, Weymark (1987, Theorem 1) has shown that an increase in the producer price of the consumption good leads to a reduction in each person's consumption. Because preferences are quasilinear, there are no income effects associated with a change in $p$. Hence, as the opportunity cost of consumption in terms of leisure increases, it is optimal to economize on consumption. The situation is more complex in an economy with a public good for two reasons. First, an increase in the price of private consumption makes the public good relatively less expensive. At the same time, changes in private consumption may induce changes in the marginal valuation of the public good. Thus, the effect of an increase in $p$ on the provision of the public good is not obvious. Moreover, the effects on private consumption need to be re-examined, especially if the provision of the public good affects the marginal utility of consumption.

Proposition 5 shows that it is possible to sign the effects on the consumptions of both goods when $p$ increases provided that the signs of the change in the marginal valuation of the public good when private consumption increases are not different for the two individuals at the initial allocation.

Proposition 5. If person one's income is positive in the initial optimal allocation, then a marginal increase in the price $p$ of the private good results in

(i) a decrease in each person's optimal consumption of the private good and a decrease in the optimal provision of the public good if the marginal valuation of the public good is nondecreasing in private consumption at the initial allocation for both individuals and it is strictly increasing for at least one individual,

(ii) a decrease in each person's optimal consumption of the private good and an increase in the optimal provision of the public good if the marginal valuation of the public good is nonincreasing in private consumption at the initial allocation for both individuals and it is strictly decreasing for at least one individual, and

(iii) a decrease in each person's optimal consumption of the private good and no change in the optimal provision of the public good if the marginal valuation of the public good is independent of private consumption at the initial allocation for both individuals.

In each of the three cases considered, an increase in the price of the private good results in a decrease in each person's optimal private consumption, just like in the model without the public good. Thus, the direct effect on private consumption of increasing the cost of the private good dominates any indirect effects caused by a change in the 
provision of the public good. Consider changing the private consumptions to their new lower optimal levels holding the quantity of the public good fixed. From (3.11), because the social marginal cost of the public good has not changed, we see that it is necessary to adjust $g$ so as to restore the social marginal benefit of the public good to its original level. If, for example, the change in the marginal valuation of the public good induced by the reduction in private consumption is negative for both individuals, $g$ must be adjusted so as to increase the social marginal benefit of the public good. Because $v$ is strictly concave, this requires decreasing the provision of the public good. If these marginal valuations move in opposite directions, the overall effect on the social marginal benefit of the public good from reducing the private consumptions is indeterminate.

Proposition 5 is silent not only about the effects of a change in $p$ on the optimal provision of the public good, but also about the implications for the optimal values of the private consumptions if the sign of the effect of an increase in private consumption on the marginal valuation of the public good differs between the two individuals. The reason for this ambiguity is clear from (5.1). For each individual $i$, because $\beta_{i}$ and $\gamma$ are constant on the right-hand side of (5.1), the changes in the optimal allocation of public and private goods owing to an increase in $p$ must result in an increase in the marginal utility of private consumption for both individuals. There are two means by which $v_{c_{i}}$ can be increased: (i) by a decrease in private consumption or (ii) by a change in public good provision, the sign of which depends on the sign of $v_{c_{i} g}$ at the intial allocation. Suppose, for the sake of argument, that $v_{c_{1} g}<0$ and $v_{c_{2} g}>0$ at the initial allocation, and that an increase in $p$ leads to a reduction in $c_{1}$. This results in a higher valuation of the public good by person one, which, all else equal, would tend to increase the optimal level of public good provision. This, in turn, results in an increase in the marginal valuation of consumption for person two. This increase may be of sufficient magnitude to ensure that (5.1) holds without a decrease in $c_{2}$.

As Weymark (1987) has shown, even without the public good, it is not possible to sign the effects of a change in $p$ on the before-tax incomes. The situation does not improve with the presence of a public good. Nor is it possible to sign what happens to person one's marginal tax rate when $p$ increases. Such a change increases the numerator in the fraction that appears in (3.12). However, as we have just seen, person one's marginal utility of consumption also increases, thereby increasing the denominator in this fraction. As a consequence, the overall effect on his marginal tax rate is indeterminate.

\subsection{Changing the Preference Parameter}

Except for the last term in the reduced-form objective function (3.4), the taste parameter $\gamma$ and the price $p$ of the private good play symmetric roles. For this reason, in the absence of the public good, Weymark (1987) found that the comparative static effects on consumption are the same for both $\gamma$ and $p$. However, the presence of the public good introduces an asymmetry between these two parameters, and this makes the analysis more complex. When $p$ increases, only the the cost of producing the private good increases. 
However, when $\gamma$ increases, the production of both goods becomes more onerous.

Proposition 6 summarizes our findings about the effects of an increase in $\gamma$ on the optimal allocation.

Proposition 6. If person one's income is positive in the initial optimal allocation, then a marginal increase in the preference parameter $\gamma$ results in a decrease in each person's optimal consumption of the private good, a decrease in the optimal provision of the public good, and a decrease in the optimal aggregate before-tax income if the marginal valuation of the public good is nondecreasing in private consumption at the initial allocation for both individuals.

As (5.1) indicates, each person's marginal utility of private consumption must increase when $\gamma$ increases, just as is the case when $p$ increases. But now we must also increase the social marginal benefit of the public good in order to satisfy (3.11), for which a sufficient condition is that each person's marginal valuation of the public good increases.

First, consider the case in which the marginal valuations of the public good are independent of private consumption for both individuals. In order to increase the social marginal benefit of the public good, it is then necessary to decrease its provision. By Young's Theorem, this has no effect on either individual's marginal utilty of private consumption. Hence, to increase these marginal utilities, the private consumptions must decrease as well.

If these marginal valuations are both increasing in private consumption, decreasing the quantity of the public good lowers the marginal utilities of private consumption, thereby reinforcing the need to decrease both private consumptions in order to increase their social marginal values. Similarly, decreasing the consumptions of the private good reinforces the need to decrease the provision of the public good so as to increase its social marginal value. On the other hand, if these marginal valuations are both decreasing in private consumption, then the indirect effect of decreasing $c_{i}$ (resp. $g$ ) on $v_{g}$ (resp. $v_{c_{i}}$ ) is positive, as required. But then it is indeterminate whether a decrease in $g$ (resp. $v_{c_{i}}$ ) is still needed for the overall effect on $v_{g}$ (resp. $v_{c_{i}}$ ) to be positive.

For the case considered in Proposition 6, it is optimal to reduce the production of both the private and public goods. Because the parameters of the technology are being held fixed, in order to keep the production-feasibility constraint binding, it is therefore necessary to reduce the aggregate labour supply in efficiency units; i.e., the sum of the before-tax incomes must decrease. However, it is not possible to determine whether both individuals reduce their labour supply or not.

The parameters $\gamma$ and $p$ enter symmetrically into the formulae for the marginal tax rates in (3.12). Thus, the reasoning used to show why the effect of an increase in $p$ on person one's marginal tax rate is indeterminate also applies for an increase in $\gamma$. 


\section{Comparative Statics for the Skill Levels and Welfare Weights}

In this section, we investigate the comparative static properties of changes in the skill levels and the relative social welfare weights. From the definitions of the adjusted welfare weights (3.5) and (3.6), the first-order conditions for the reduced-form problem (3.10) and (3.11), and the expressions for the optimal incomes (3.8) and (3.9), it can be seen that the effects of these parameters are mediated through their effects on the adjusted welfare weights $\beta_{1}$ and $\beta_{2}$, with changes in person's one's skill level and in the relative welfare weights only affecting $\beta_{1}$ and changes in person two's skill level afffecting both $\beta_{1}$ and $\beta_{2}$. We begin by considering the parameters that only influence the optimal allocation through their effects on $\beta_{1}$.

\subsection{Changing Person One's Skill Level and the Relative Welfare Weights}

As a preliminary step in our analysis, we determine what can be said about the comparative static properties of $\beta_{1}$ without knowing whether the change in $\beta_{1}$ is due to a change in $w_{1}$ or $\lambda_{1}{ }^{17}$ If there is no public good, Weymark (1987, Proposition 5) has shown that an increase in an individual's adjusted welfare weight increases that person's optimal consumption, but does not affect anybody else's consumption. The reason is that the terms containing the adjusted welfare weights in the first-order conditions for private consumption are independent of each other. However, in the presence of a public good, these expressions may not be independent because, as (3.10) demonstrates, the quantity of the public good may affect everyone's marginal utility of private consumption. As a consequence, the comparative static analysis of an increase in person one's adjusted welfare weight is more complex when there is a public good than when there is not.

The comparative static properties of an increase in $\beta_{1}$ are presented in Proposition 7.

Proposition 7. If person one's income is positive and his marginal valuation of the public good is nondecreasing in private consumption at the initial optimal allocation, then a marginal increase in person one's adjusted welfare weight $\beta_{1}$ results in

(i) an increase in the optimal provision of the public good,

(ii) an increase in person one's optimal consumption of the private good,

(iii) an increase in person two's optimal consumption of the private good if and only if person two has a marginal valuation of the public good that is increasing in private consumption at the initial allocation, and

\footnotetext{
${ }^{17}$ Recall that the welfare weights have been normalized so that $\lambda_{2}$ is completely determined by $\lambda_{1}$. Because $\beta_{2}=w_{2}$ and $w_{2}$ also helps determine the value of $\beta_{1}$, it is not possible to change $\beta_{2}$ without also changing the value of $\beta_{1}$. Therefore, except in the proof of Proposition 9, we do not consider the comparative statics of an independent change in $\beta_{2}$.
} 
(iv) an increase in the optimal aggregate before-tax income if person two has a marginal valuation of the public good that is nondecreasing in private consumption at the initial allocation.

An increase in the adjusted welfare weight $\beta_{1}$ of person one has no effect on the social marginal cost of either the private or public good in the reduced-form problem. Furthermore, at the initial allocation, raising $\beta_{1}$ increases both the social marginal benefit of one's private consumption and of the public good, but has no effect on the social marginal benefit of person two's private consumption. Because the social marginal benefits of person one's private consumption and of the public good exceed their social marginal costs at the initial allocation, one would expect that it is optimal to increase both of these variables. Proposition 7 validates this intuition provided that person one's marginal valuation of the public good is nondecreasing in his private consumption at the initial allocation.

In order to satisfy the first-order conditions (3.10) and (3.11), it is necessary to reduce person one's marginal utility of consumption and hold constant person two's marginal utility of consumption when $\beta_{1}$ is increased. It is also necessary to adjust the two individuals' marginal valuations of the public good so as to restore the social marginal benefit of the public good to its original value. If the marginal valuations of the public good are independent of private consumption for both individuals, the only way to achieve these objectives is to increase both $c_{1}$ and $g$ and to not change $c_{2}$.

If, however, the marginal valuations of the public good are not independent of private consumption, we also need to take account of the indirect effects of changing $c_{i}$ (resp. $g$ ) on $v_{g_{i}}$ (resp. $v_{c_{i}}$ ). If $v_{c_{1} g}$ is positive, raising $g$ increases $v_{c_{1}}$, which necessitates an increase in $c_{1}$ in order to lower this marginal utility below its original value. On the other hand, if contrary to our assumption, $v_{c_{1} g}$ is negative, then raising $g$ decreases $v_{c_{1}}$, and it is no longer clear whether we need to increase or decrease $c_{1}$ in order to equate the social marginal benefit and social marginal cost of one's private consumption. These conclusions are reinforced by considering the effects of increasing $c_{1}$ on the optimal provision of the public good. If $v_{c_{1} g}$ is positive, raising $c_{1}$ further increases the social marginal benefit of the public good, which, for fixed $c_{2}$, requires increasing $g$ to restore the equality between the social marginal benefit and the social marginal cost of the public good. On the other hand, if $v_{c_{1} g}$ is negative, it is not clear how $g$ must be changed to restore this equality.

Of course, we also need to consider possible changes in the value of $c_{2}$. Proposition 7 shows that the indirect effects of changing $c_{2}$ are never strong enough to reverse our conclusion that $g$ should be increased. Because $g$ is increased, in order to offset its effect on $v_{c_{2}}, c_{2}$ must be increased if and only if the marginal utility of person two's private consumption is increasing in $g$, which, by Young's Theorem, is equivalent to the statement in part (iii) of the Proposition.

For the case considered in part (iv), it is optimal to increase the production of both the private and the public good. Because the parameters of the technology have not changed, this necessitates an increase in the supply of labour in efficiency units or, equivalently, 
an increase in the sum of the before-tax incomes.

Because $\beta_{2}=w_{2}$, from the definition of $\beta_{1}$ in (3.5), we see that an increase in $w_{1}$ or a decrease in $\lambda_{1}$ (with a compensating increase in $\lambda_{2}$ to keep their sum constant) leads to an increase in $\beta_{1}$, but does not affect the value of $\beta_{2}$. Moreover, changes in $w_{1}$ or $\lambda_{1}$ only influence the optimal consumptions of the private and public goods through the effects they have on $\beta_{1}$. Thus, all of the comparative static results presented in Proposition 7 also hold for an increase in $w_{1}$ or a decrease in $\lambda_{1}$.

When the increase in $\beta_{1}$ is due solely to a decrease in $\lambda_{1}$, we can say somewhat more. From the first-order condition (3.10), we know that $v_{c_{1}}$ must decrease to compensate for the increase in $\beta_{1}$. Thus, from (3.12), person one's marginal tax rate must decrease. ${ }^{18}$ The set of feasible utility pairs $\left(u_{1}, u_{2}\right)$ does not depend on the welfare weights. With a weighted utilitarian social welfare function, it cannot be optimal to make person one better off if the relative weight on his utility is decreased. When $\lambda_{1}$ is decreased, we know that it is optimal to increase person one's consumption of both goods if his marginal valuation of the public good is nondecreasing in private consumption at the initial optimal allocation. Thus, in order not to increase his utility, he must work more; i.e., his before-tax income must increase. The effect of changing $\lambda_{1}$ on person two's income is ambiguous. Person two must be no worse off, but because his consumption of the public good increases, changes in either direction in his private consumption and before-tax income are compatible with an increase his utility. ${ }^{19}$

The preceding discussion is summarized in Proposition 8.

Proposition 8. If person one's income is positive and his marginal valuation of the public good is nondecreasing in private consumption at the initial optimal allocation, then the comparative static results presented in Proposition 7 for a marginal increase in $\beta_{1}$ also hold for a marginal increase in person one's skill level $w_{1}$ or a marginal decrease in his welfare weight $\lambda_{1}$. Furthermore, a marginal decrease in $\lambda_{1}$ results in

(i) a decrease in person one's marginal tax rate and

(ii) an increase in person one's optimal before-tax income if his marginal valuation of the public good is nondecreasing in private consumption at the initial optimal allocation.

\subsection{Changing Person Two's Skill Level}

Because it is optimal for the incentive-compatibility constraint of person two, but not person one, to bind, the two skill levels, $w_{1}$ and $w_{2}$, enter asymmetrically in the reducedform objective function (3.4); $w_{1}$ only affects the value of $\beta_{1}$, whereas $w_{2}$ affects the values

\footnotetext{
${ }^{18}$ If $w_{1}$ increases, it is not possible to sign the direction of change in this marginal tax rate because the two terms in the denominator of the fraction in (3.12) move in opposite directions.

${ }^{19}$ If there is no public good, as previously noted, when $\beta_{1}$ is increased, it is optimal not to change person two's consumption. Thus, as Weymark (1987) has formally shown, to make person two better off, his before-tax income must fall.
} 
of both $\beta_{1}$ and $\beta_{2}$. As can be seen from (3.5) and (3.6), a change in $w_{2}$ moves $\beta_{1}$ and $\beta_{2}$ in opposite directions, rendering the effects of a change in $w_{2}$ on the optimal allocation difficult to sign. A simple description of the comparative static properties of $w_{2}$ only appears to be available for the special case in which private and public consumption are additively separable in the utility function. Proposition 9 presents our comparative static results for a change in person two's skill level for this special case.

Proposition 9. If person one's income is positive and the function $v$ is additively separable, then a marginal increase in person two's skill level $w_{2}$ results in

(i) an increase in the optimal provision of the public good,

(ii) a decrease in person one's optimal consumption of the private good,

(iii) an increase in person two's optimal consumption of the private good, and

(iv) an increase in person one's marginal tax rate.

With additively separable preferences, the consumption of the public good has no effect on the marginal utility of private consumption. Because an increase in $w_{2}$ decreases $\beta_{1}$ (resp. increases $\beta_{2}$ ), it is necessary to decrease $c_{1}$ (resp. increase $c_{2}$ ) in order to raise $v_{c_{1}}$ (resp. lower $v_{c_{2}}$ ) so that the social marginal benefits of these goods are held constant, as required by the first-order condition (3.10). Because $p, \gamma$, and $w_{1}$ are being held fixed, increasing $v_{c_{1}}$ also increases person one's marginal tax rate.

The intuition for the effect of a change on $w_{2}$ on the optimal provision of the public good is less straightforward. Because private consumption has no effect on the marginal valuations of the public good, the level of provision of this good is the only instrument available to keep the marginal social benefit of the public good from changing when $w_{2}$ is increased, as required by the first-order condition (3.11). Because both individuals have the same additively-separable utility function for the two consumption goods, they also have the same marginal valuation of the public good. As a consequence, the provision of the public good should be increased if and only if the sum $\beta_{1}+\beta_{2}$ of the adjusted welfare weights is increased. Because $\partial \beta_{1} / \partial w_{2}=1-\lambda_{1}$ and $\partial \beta_{2} / \partial w_{2}=1$, an increase in $w_{2}$ increases the overall social marginal value of the public good. Thus, provision of the public good should be increased.

It is clear from the preceding discussion that the additive separability of $v$ plays an important role in establishing part (i) of Proposition 9. For more general utility functions, even if the results described in parts (ii) and (iii) continue to hold, the net effect of changing the private consumptions on the social marginal valuation of the public good is difficult to sign, even in the absence of the resulting feedbacks on the marginal utility of consumption.

Even with additively separable preferences, it is not possible to determine how the before-tax incomes should change when $w_{2}$ is increased. Parts (ii) and (iii) of Proposition 9 together with the separability assumption imply that the last term in (3.8) and (3.9) 
increases. Part (i) of Proposition 9 implies that the second term in these expressions also increases. However, because it is optimal to move $c_{1}$ and $c_{2}$ in opposite directions, the sign of the first term is indeterminate.

\section{Concluding Remarks}

A natural extension of our analysis would be to consider more types of individuals. Weymark (1987) has provided fairly extensive comparative static results for an arbitrary finite number of skill levels when there is no public good. There are grounds for believing that some unambiguous comparative statics can also be obtained for a multi-type version of our model. With more types, it remains optimal for the production-feasibility constraint to bind and, if the welfare weights are declining in skill level, it is also optimal for all the adjacent downward self-selection constraints to bind. This permits us to solve for the optimal values of the private and public goods using a reduced-form optimization problem. The objective function in this problem has the same general form as here; we simply add a social benefit term for each additional type and replace $c_{1}+c_{2}$ with the sum of the individual private consumptions. An individual's adjusted welfare weight would now depend on the sum of his welfare weight and the welfare weights of all individuals of lower ability. Provided that there is no bunching at the initial optimal allocation, the first-order optimality conditions for private consumption have the same form as in (3.10), and the first-order optimality condition for public good provision has an additional term for each additional type.

The comparative static properties of our model for the consumptions of the private and public goods are contained in the comparative static matrix $A^{-1} B$ given in (4.8). Except for the last row and column, the $A$ matrix only has non-zero entries on the main diagonal. Similarly, the $B$ matrix contains a submatrix that only has non-zero entries on its main diagonal. These properties of $A$ and $B$ carry over to their multi-type analogues. It is the sparseness of these matrices that suggests that some of our results can be generalized to more skill types.

Although our theorems carry over quite straightforwardly to an arbitrary number of individuals of each type, by not explicitly including the number of each type in our model, we are unable to consider how the optimal allocation or the marginal tax rate of the low-skilled individuals varies with respect to a change in the distribution of types. As noted in Section 1, in models without a public good, Hamilton and Pestieau (2002) and Boadway and Pestieau (2004) have analyzed the impact of changing the skill distribution on, respectively, the individual utilities and the utility possibilities frontier. It would be of interest to see how changing the proportions of each type affects the optimal allocation and the optimal marginal tax rates both with and without public good provision.

An obvious limitation of our analysis is the restriction to quasilinear preferences. If the two consumption goods are not separable from labour in the utility function, then the Samuelson Rule for the optimal provision of the public good no longer holds. However, before attempting to generalize our model to non-separable preferences, it seems prudent 
to first try to obtain comparative static results for a non-separable preference version of the private good model considered by Weymark (1987). When the social welfare function is an increasing, symmetric, and concave function of the individual utilities, Röell (1985) has shown that it is optimal for the downward self-selection constraints to bind. It is also optimal for the production-feasibility constraint to bind. Thus, in principle, it is possible to use these binding constraints to solve for the optimal incomes as a function of the optimal consumptions, and thereby obtain a reduced-form problem to solve for the optimal consumptions. The resulting reduced-form objective function would not, in general, be additively separable in the consumption variables, which would preclude obtaining explicit solutions for the optimal consumptions in terms of the underlying parameters of the model, as in Weymark (1987). Nevertheless, techniques similar to those employed here could be used to see if the first-order conditions of the reduced-form problem yield any unambiguous comparative statics.

\section{Appendix}

Proof of Lemma 1. (i) We first show that the production-feasibility contraint binds. By assumption, $\tilde{a}=\left(\tilde{y}_{1}, \tilde{y}_{2}, \tilde{c}_{1}, \tilde{c}_{2}, \tilde{g}\right)$ satisfies (2.3). Suppose, contrary to (3.1), that

$$
p\left(\tilde{c}_{1}+\tilde{c}_{2}\right)+q \tilde{g}<\tilde{y}_{1}+\tilde{y}_{2} .
$$

Because $\tilde{y}_{1}>0$, it follows from (2.12) that $\tilde{y}_{2}>0$. Hence, there then exists a $\delta>0$ such that

$$
p\left(\tilde{c}_{1}+\tilde{c}_{2}\right)+q \tilde{g} \leq\left(\tilde{y}_{1}-\delta\right)+\left(\tilde{y}_{2}-\delta\right) .
$$

Consider the allocation $\hat{a}=\left(\hat{y}_{1}, \hat{y}_{2}, \hat{c}_{1}, \hat{c}_{2}, \hat{g}\right)=\left(\tilde{y}_{1}-\delta, \tilde{y}_{2}-\delta, \tilde{c}_{1}, \tilde{c}_{2}, \tilde{g}\right)$. By (A.2), $\hat{a}$ satisfies (2.3). Because $\tilde{a}$ satisfies (2.9) and (2.10), by (2.6), $\hat{a}$ also satisfies these two constraints. Hence, by (2.6) and (2.15),

$$
\mathcal{W}(\hat{a})-\mathcal{W}(\tilde{a})=\left(\lambda_{1}+\lambda_{2}\right) \delta>0,
$$

which contradicts the optimality of $\tilde{a}$.

(ii) Next, we show that the higher-productivity individual's self-selection constraint binds. By assumption, $\tilde{a}$ satisfies (2.10). Suppose, by way of contradiction to (3.2), that

$$
w_{2} v\left(\tilde{c}_{2}, \tilde{g}\right)-\gamma \tilde{y}_{2}>w_{2} v\left(\tilde{c}_{1}, \tilde{g}\right)-\gamma \tilde{y}_{1}
$$

Because $\tilde{y}_{1}>0$, there exists an $\varepsilon>0$ such that

$$
w_{2} v\left(\tilde{c}_{2}, \tilde{g}\right)-\gamma\left(\tilde{y}_{2}+\varepsilon\right) \geq w_{2} v\left(\tilde{c}_{1}, \tilde{g}\right)-\gamma\left(\tilde{y}_{1}-\varepsilon\right) .
$$

Consider the allocation $\hat{a}=\left(\hat{y}_{1}, \hat{y}_{2}, \hat{c}_{1}, \hat{c}_{2}, \hat{g}\right)=\left(\tilde{y}_{1}-\varepsilon, \tilde{y}_{2}+\varepsilon, \tilde{c}_{1}, \tilde{c}_{2}, \tilde{g}\right)$. Because utility is decreasing in income and $\tilde{a}$ satisfies (2.9), so does $\hat{a}$. Because $\tilde{a}$ satisfies (2.3) and total income is unchanged, $\hat{a}$ also satisfies (2.3). By (2.6) and (2.15),

$$
\mathcal{W}(\hat{a})-\mathcal{W}(\tilde{a})=\left(\lambda_{1}-\lambda_{2}\right) \varepsilon>0,
$$


which contradicts the optimality of $\tilde{a}$.

(iii) While it is possible to provide a direct proof that the low-productivity individual's self-selection contraint does not bind, an indirect proof is simpler. In the course of proving Proposition 1, we show that (3.3) holds. The no-bunching result follows immediately from (3.2) and (3.3).

Proof of Lemma 2. Because the partial derivatives of $v$ approach infinity as either argument goes to zero, if $\left(\tilde{c}_{1}, \tilde{c}_{2}, \tilde{g}\right)$ solves the reduced-form problem, then $\tilde{c}_{1}, \tilde{c}_{2}$, and $\tilde{g}$ are positive. Because the reduced-form objective function is strictly concave on $\mathbb{R}_{++}^{2}$ and continuous on $\mathbb{R}_{+}^{2}$, the partial derivatives of $v$ approach zero as either argument goes to infinity, and the constraint set in the reduced-form optimization problem is defined by linear inequalities, this problem has a unique solution. An immediate implication of the fact that $\beta_{2}>\beta_{1}$ is that the monotonicity constraint (2.11) does not bind at the optimum.

Proof of Lemma 3. Because $a=\left(y_{1}, y_{2}, c_{1}, c_{2}, g\right)$ satisfies (3.2),

$$
w_{2} v\left(c_{2}, g\right)=w_{2} v\left(c_{1}, g\right)-\gamma y_{1}+\gamma y_{2}
$$

Thus, by (2.6) and (2.15),

$$
\mathcal{W}(a)=\lambda_{1} w_{1} v\left(c_{1}, g\right)-\lambda_{1} \gamma y_{1}+\lambda_{2} w_{2} v\left(c_{1}, g\right)-\lambda_{2} \gamma y_{1}
$$

Using the normalization in $(2.16)$, (A.8) can be rewritten as

$$
\mathcal{W}(a)=\lambda_{1} w_{1} v\left(c_{1}, g\right)+\left(1-\lambda_{1}\right) w_{2} v\left(c_{1}, g\right)+w_{2} v\left(c_{1}, g\right)-2 \gamma y_{1} .
$$

Note that

$$
\beta_{1}=\lambda_{1} w_{1}+\left(1-\lambda_{1}\right) w_{2}
$$

Hence, (A.9) implies that

$$
\mathcal{W}(a)=\beta_{1} v\left(c_{1}, g\right)+w_{2} v\left(c_{1}, g\right)-2 \gamma y_{1}
$$

Using (A.7) in (A.11), it follows that

$$
\mathcal{W}(a)=\beta_{1} v\left(c_{1}, g\right)+w_{2} v\left(c_{2}, g\right)-\gamma y_{1}-\gamma y_{2} .
$$

Because $a$ satisfies (3.1), (A.12) implies that

$$
\mathcal{W}(a)=\beta_{1} v\left(c_{1}, g\right)+\beta_{2} v\left(c_{2}, g\right)-\gamma p\left(c_{1}+c_{2}\right)-\gamma q g
$$

where use has been made of the fact that $\beta_{2}=w_{2}$. Thus, $\mathcal{W}(a)=W\left(c_{1}, c_{2}, g\right)$, as required. 
Proof of Proposition 1. First note that (3.2) can be rewritten as

$$
\tilde{y}_{2}=\tilde{y}_{1}+\frac{1}{\gamma} w_{2}\left[v\left(\tilde{c}_{2}, \tilde{g}\right)-v\left(\tilde{c}_{1}, \tilde{g}\right)\right]
$$

By adding the expressions in (3.8) and (3.9), it is then clear that these equalities are equivalent to (3.1) and (3.2).

Suppose that $\tilde{a}=\left(\tilde{y}_{1}, \tilde{y}_{2}, \tilde{c}_{1}, \tilde{c}_{2}, \tilde{g}\right)$ is the allocation identified in part (ii) of Proposition 1. The preceding discussion shows that the production-feasibility constraint and person two's incentive-compatibility constraint both bind. From Lemma 2, we know that $\tilde{c}_{2}>\tilde{c}_{1}$ at a solution to the reduced-form problem. Because $v$ is increasing in its first argument, the income in (3.9) is larger than the income in (3.8). Single-crossing then implies that $\tilde{a}$ satisfies person one's incentive-compatibility constraint with a strict inequality. Thus, $\tilde{a}$ satisfies all of the constraints of the optimal nonlinear income tax problem. The equivalence of the allocations in parts (i) and (ii) of Proposition 1 now follows from Lemma 3 and the two equalities in Lemma 1.

Proof of Proposition 2. (i) It follows from (3.10) that

$$
\frac{\gamma p}{\beta_{i} v_{c_{i}}\left(\tilde{c}_{i}, \tilde{g}\right)}=1, \quad i=1,2
$$

Because $\beta_{1}<w_{1},(3.12)$ and (A.15) imply that

$$
\operatorname{MTR}^{1}\left(\tilde{y}_{1}, \tilde{c}_{1}, \tilde{g}\right)=1-\gamma p / w_{1} v_{c_{1}}\left(\tilde{c}_{1}, \tilde{g}\right)>1-\gamma p / \beta_{1} v_{c_{1}}\left(\tilde{c}_{1}, \tilde{g}\right)=0 .
$$

Because all the quantities in the second term on the right-hand side of (3.12) are positive, (A.16) establishes (3.13).

(ii) By definition, $\beta_{2}=w_{2}$. Hence, (3.14) follows directly from (3.12) and (A.15).

(iii) It follows from (3.10) that

$$
\beta_{i} v_{c_{i}}\left(\tilde{c}_{i}, \tilde{g}\right)=\gamma p, \quad i=1,2 .
$$

Dividing each term in (3.11) by $\gamma p$ and using (A.17) implies that

$$
\frac{\beta_{1} v_{g}\left(\tilde{c}_{1}, \tilde{g}\right)}{\beta_{1} v_{c_{1}}\left(\tilde{c}_{1}, \tilde{g}\right)}+\frac{\beta_{2} v_{g}\left(\tilde{c}_{2}, \tilde{g}\right)}{\beta_{2} v_{c_{2}}\left(\tilde{c}_{2}, \tilde{g}\right)}-\frac{\gamma q}{\gamma p}=0 .
$$

Using (2.8) in (A.18) and simplifying, we obtain (3.15).

Proof of Proposition 3. Implicit differentiation of (3.10) and (3.11) yields

$$
A\left[d c_{1}, d c_{2}, d g\right]^{\top}=B\left[d q, d p, d \gamma, d \beta_{1}, d \beta_{2}\right]^{\top} .
$$

Because $v$ is twice continuously differentiable, the middle expressions of (3.10) and (3.11) are continuously differentiable functions of the eight variables $c_{1}, c_{2}, g, q, p, \gamma, \beta_{1}$, and $\beta_{2}$. 
Consider any $\left(\bar{q}, \bar{p}, \bar{\gamma}, \bar{\beta}_{1}, \bar{\beta}_{2}\right) \in \mathbb{R}_{++}^{5}$. Provided that $A$ is invertible at $\left(\bar{q}, \bar{p}, \bar{\gamma}, \bar{\beta}_{1}, \bar{\beta}_{2}\right)$, it follows from the Implicit Function Theorem that there exists a neighbourhood $N$ contained in $\mathbb{R}_{++}^{5}$ of $\left(\bar{q}, \bar{p}, \bar{\gamma}, \bar{\beta}_{1}, \bar{\beta}_{2}\right)$ and a unique continuously differentiable function $\bar{F}: N \rightarrow$ $\mathbb{R}_{++}^{3}$ mapping $\left(q, p, \gamma, \beta_{1}, \beta_{2}\right)$ to $\left(c_{1}, c_{2}, g\right)$ for which $\left(\bar{F}\left(q, p, \gamma, \beta_{1}, \beta_{2}\right), q, p, \gamma, \beta_{1}, \beta_{2}\right)$ solves (3.10) and (3.11) for all $\left(q, p, \gamma, \beta_{1}, \beta_{2}\right) \in N .{ }^{20}$ Because the function $\bar{F}$ is unique, it must therefore coincide with $F$ on $N$. Thus, to show that $F$ is continuously differentiable, it only remains to show that $A$ is invertible at any $\left(q, p, \gamma, \beta_{1}, \beta_{2}\right) \in \mathbb{R}_{++}^{5}$.

In order to establish that $A$ is invertible, it suffices to show that $A$ has a non-zero determinant. Performing the Laplace expansion along the first row of $A$ yields

$$
\begin{aligned}
|A| & =\beta_{1} v_{c_{1} c_{1}}\left[\beta_{2} v_{c_{2} c_{2}}\left(\beta_{1} v_{g_{1} g_{1}}+\beta_{2} v_{g_{2} g_{2}}\right)-\left(\beta_{2} v_{c_{2} g}\right)^{2}\right]+\beta_{1} v_{c_{1} g}\left[-\beta_{1} \beta_{2} v_{c_{1} g} v_{c_{2} c_{2}}\right] \\
& =\beta_{1} \beta_{2}^{2} v_{c_{1} c_{1}}\left[v_{c_{2} c_{2}} v_{g_{2} g_{2}}-\left(v_{c_{2} g}\right)^{2}\right]+\beta_{1}^{2} \beta_{2} v_{c_{2} c_{2}}\left[v_{c_{1} c_{1}} v_{g_{1} g_{1}}-\left(v_{c_{1} g}\right)^{2}\right] .
\end{aligned}
$$

Strict concavity of $v$ implies that each term in square brackets in the second line of (A.20) is positive and that $v_{c_{i} c_{i}}<0$ for $i=1,2$. Thus, $|A|<0$.

Multiplying both sides of (A.19) by $A^{-1}$ yields (4.2).

Proof of Proposition 4. By the final row of (4.9),

$$
\frac{\partial g^{*}}{\partial q}=\frac{\gamma \beta_{1} \beta_{2} v_{c_{1} c_{1}} v_{c_{2} c_{2}}}{|A|} .
$$

Strict concavity of $v$ ensures that the numerator on the right-hand side of (A.21) is positive. In the proof of Proposition 3, we have shown that $|A|$ is negative. Thus, $\partial g^{*} / \partial q<0$, which establishes part (i) of the Proposition.

The first and second rows of (4.9) show that

$$
\frac{\partial c_{i}^{*}}{\partial q}=-\frac{\gamma \beta_{1} \beta_{2} v_{c_{j} c_{j}}}{|A|} v_{c_{i} g}, \quad i, j=1,2, i \neq j .
$$

By the strict concavity of $v, v_{c_{i} c_{i}}<0$ for $i=1,2$. Because $|A|<0$, the fraction that multiplies $v_{c_{i} g}$ on the right-hand side of (A.22) is positive. Hence, $\partial c_{i}^{*} / \partial q$ has the opposite sign as $v_{c_{i} g}$, which establishes part (ii) of the Proposition.

The chain rule and (4.9) imply that at $\left(c_{1}^{*}\left(q, p, \gamma, \beta_{1}, \beta_{2}\right), g^{*}\left(q, p, \gamma, \beta_{1}, \beta_{2}\right)\right)$,

$$
\begin{aligned}
\frac{\partial v_{c_{1}}}{\partial q} & =v_{c_{1} c_{1}} \frac{\partial c_{1}^{*}}{\partial q}+v_{c_{1} g} \frac{\partial g^{*}}{\partial q} \\
& =\frac{1}{|A|}\left(-v_{c_{1} c_{1}} \gamma \beta_{1} \beta_{2} v_{c_{2} c_{2}} v_{c_{1} g}+v_{c_{1} g} \gamma \beta_{1} \beta_{2} v_{c_{1} c_{1}} v_{c_{2} c_{2}}\right)=0 .
\end{aligned}
$$

It then follows from (A.23) and (3.12) that the marginal tax rate faced by person one is invariant to the increase in $q$, which establishes part (iii) of the Proposition.

\footnotetext{
${ }^{20}$ See Spivak (1965, Theorem 2-12) for a statement of the Implicit Function Theorem that is general enough for our purposes.
} 
Proof of Proposition 5. By (4.10) and the definition of $\mu$, we have

$$
\begin{aligned}
& \frac{\partial c_{1}^{*}}{\partial p}=\frac{\gamma\left\{\beta_{2}^{2}\left[v_{c_{2} c_{2}} v_{g_{2} g_{2}}-\left(v_{c_{2} g}\right)^{2}\right]+\beta_{1} \beta_{2} v_{c_{2} c_{2}} v_{g_{1} g_{1}}+\beta_{1} \beta_{2} v_{c_{1} g} v_{c_{2} g}\right\}}{|A|}, \\
& \frac{\partial c_{2}^{*}}{\partial p}=\frac{\gamma\left\{\beta_{1}^{2}\left[v_{c_{1} c_{1}} v_{g_{1} g_{1}}-\left(v_{c_{1} g}\right)^{2}\right]+\beta_{1} \beta_{2} v_{c_{1} c_{1}} v_{g_{2} g_{2}}+\beta_{1} \beta_{2} v_{c_{1} g} v_{c_{2} g}\right\}}{|A|},
\end{aligned}
$$

and

$$
\frac{\partial g^{*}}{\partial p}=-\frac{\gamma\left\{\beta_{1} \beta_{2} v_{c_{1} c_{1}} v_{c_{2} g}+\beta_{1} \beta_{2} v_{c_{2} c_{2}} v_{c_{1} g}\right\}}{|A|} .
$$

By the strict concavity of $v$, each of the first two terms in braces on the right-hand sides of (A.24) and (A.25) are positive. In each of the three cases considered in the Proposition, the last terms in these braces are nonnegative. Thus, because $|A|<0$, (A.24) and (A.25) imply that the optimal private consumption of each individual falls when $p$ increases in all three parts of the Proposition.

In part (i) of the Proposition, both $v_{c_{1} g}$ and $v_{c_{2} g}$ are nonnegative and at least one of these partial derivatives is positive. Thus, both terms in braces in (A.26) are nonpositive and at least one of these terms is negative. Because $|A|<0$, it then follows from (A.26) that the optimal provision of the public good decreases when $p$ increases.

Simply replacing "nonpositive" with "nonnegative" and "positive" with "negative" in the preceding argument shows that the optimal provision of the public good increases when $p$ increases in part (ii) of the Proposition.

In part (iii) of the Proposition, both $v_{c_{1} g}=0$ and $v_{c_{2} g}=0$. Thus, the numerator on the right-hand side of (A.26) vanishes and the optimal provision of the public good does not change when $p$ increases.

Proof of Proposition 6. By (4.11) and the definition of $\mu$, we have

$$
\begin{aligned}
\frac{\partial c_{1}^{*}}{\partial \gamma} & =\frac{p\left\{\left[\beta_{2}^{2}\left[v_{c_{2} c_{2}} v_{g_{2} g_{2}}-\left(v_{c_{2} g}\right)^{2}\right]+\beta_{1} \beta_{2}\left(v_{c_{2} c_{2}} v_{g_{1} g_{1}}+v_{c_{1} g} v_{c_{2} g}\right)\right\}-q \beta_{1} \beta_{2} v_{c_{2} c_{2}} v_{c_{1} g}\right.}{|A|}, \\
\frac{\partial c_{2}^{*}}{\partial \gamma} & =\frac{p\left\{\left[\beta_{1}^{2}\left[v_{c_{1} c_{1}} v_{g_{1} g_{1}}-\left(v_{c_{1} g}\right)^{2}\right]+\beta_{1} \beta_{2}\left(v_{c_{1} c_{1}} v_{g_{2} g_{2}}+v_{c_{1} g} v_{c_{2} g}\right)\right\}-q \beta_{1} \beta_{2} v_{c_{1} c_{1}} v_{c_{2} g}\right.}{|A|},
\end{aligned}
$$

and

$$
\frac{\partial g^{*}}{\partial \gamma}=\frac{-p\left\{\beta_{1} \beta_{2}\left(v_{c_{1} c_{1}} v_{c_{2} g}+v_{c_{2} c_{2}} v_{c_{1} g}\right)\right\}+q \beta_{1} \beta_{2} v_{c_{1} c_{1}} v_{c_{2} c_{2}}}{|A|}
$$

By the strict concavity of $v$, the first term in braces on the right-hand sides of (A.27) and (A.28) are positive and all of the own second partial derivatives of $v$ are negative. 
By assumption, $v_{c_{1} g}$ and $v_{c_{2} g}$ are nonnegative. Hence, the numerators on the right-hand sides of (A.27), (A.28), and (A.29) are all positive. Because $|A|<0$, it then follows that all three of the partial derivatives in these equations are negative.

Because the optimal values of $c_{1}, c_{2}$, and $g$ all decrease when $p$ is increased, the sum of the two before-tax incomes must also decrease in order for the production-feasibility constraint to bind.

Proof of Proposition \%. By the last row of (4.12), we have

$$
\frac{\partial g^{*}}{\partial \beta_{1}}=\frac{v_{c_{1}} \beta_{1} \beta_{2} v_{c_{2} c_{2}} v_{c_{1} g}-v_{g_{1}} \beta_{1} \beta_{2} v_{c_{1} c_{1}} v_{c_{2} c_{2}}}{|A|} .
$$

By assumption, $v_{c_{1} g} \geq 0$. Thus, the increasingness and strict concavity of $v$ imply that the first term in the numerator of the right-hand side of (A.30) is nonpositive and the second term is positive. Thus, this numerator is negative. Because $|A|<0$, it then follows from (A.30) that the optimal provision of the public good increases when $\beta_{1}$ increases, which establishes part (i) of the Proposition.

The first row of (4.12) and the definition of $\mu$ imply that

$$
\frac{\partial c_{1}^{*}}{\partial \beta_{1}}=\frac{-v_{c_{1}}\left\{\beta_{2}^{2}\left[v_{c_{2} c_{2}} v_{g_{2} g_{2}}-\left(v_{c_{2} g}\right)^{2}\right]+\beta_{1} \beta_{2} v_{c_{2} c_{2}} v_{g_{1} g_{1}}\right\}+v_{g_{1}} \beta_{1} \beta_{2} v_{c_{2} c_{2}} v_{c_{1} g}}{|A|} .
$$

Strict concavity of $v$ implies that the expression enclosed in braces on the right-hand side of (A.31) is positive. Thus, the first term in the numerator in this fraction is negative. By assumption, $v_{c_{1} g} \geq 0$ and, hence, the increasingness and strict concavity of $v$ imply that the second term in the numerator is nonpositive. Therefore, the entire numerator is negative. Because $|A|<0$, it then follows from (A.31) that person one's optimal consumption of the private good increases when $\beta_{1}$ increases, which establishes part (ii) of the Proposition.

The second row of (4.12) implies that

$$
\frac{\partial c_{2}^{*}}{\partial \beta_{1}}=\left(\frac{-v_{c_{1}} \beta_{1} \beta_{2} v_{c_{1} g}+v_{g_{1}} \beta_{1} \beta_{2} v_{c_{1} c_{1}}}{|A|}\right) v_{c_{2} g} .
$$

Because, by assumption, $v_{c_{1} g} \geq 0$, the increasingness and strict concavity of $v$ imply that the numerator in the fraction that appears on the right-hand side of (A.32) is negative. Thus, this fraction is positive because $|A|<0$. Hence, the sign of $\partial c_{2}^{*} / \partial \beta_{1}$ is the same as the sign of $v_{c_{2} g}$, which establishes part (iii) of the Proposition.

When both $v_{c_{1} g}$ and $v_{c_{2} g}$ are nonnegative, the first three parts of the Proposition imply that the optimal values of $c_{1}$ and $g$ decrease and the optimal value of $c_{2}$ does not increase when $\beta_{1}$ is increased. Because the technology parameters $p$ and $q$ are being held fixed, the sum of the two before-tax incomes must decrease in order for the production-feasibility constraint to bind, which establishes part (iv) of the Proposition. 
Proof of Proposition 9. By the chain rule and the definitions (3.5) and (3.6) of $\beta_{1}$ and $\beta_{2}$,

$$
\frac{\partial x^{*}}{\partial w_{2}}=\frac{\partial x^{*}}{\partial \beta_{1}} \frac{\partial \beta_{1}}{\partial w_{2}}+\frac{\partial x^{*}}{\partial \beta_{2}} \frac{\partial \beta_{2}}{\partial w_{2}}=\left(1-\lambda_{1}\right) \frac{\partial x^{*}}{\partial \beta_{1}}+\frac{\partial x^{*}}{\partial \beta_{2}}, \quad x=c_{1}, c_{2}, g
$$

By assumption, $v_{c_{1} g}=0$ and $v_{c_{2} g}=0$ for all values of their arguments. Hence, the last rows of (4.12) and (4.13) simplify to

$$
\frac{\partial g^{*}}{\partial \beta_{1}}=\frac{-v_{g_{1}} \beta_{1} \beta_{2} v_{c_{1} c_{1}} v_{c_{2} c_{2}}}{|A|}
$$

and

$$
\frac{\partial g^{*}}{\partial \beta_{2}}=\frac{-v_{g_{2}} \beta_{1} \beta_{2} v_{c_{1} c_{1}} v_{c_{2} c_{2}}}{|A|}
$$

Because $v$ is assumed to be additively separable and each individual enjoys the same amount of the public good, $v_{g_{1}}=v_{g_{2}}$. Hence, by (A.33), (A.34), and (A.35),

$$
\begin{aligned}
\frac{\partial g^{*}}{\partial w_{2}} & =\frac{-v_{g_{1}} \beta_{1} \beta_{2} v_{c_{1} c_{1}} v_{c_{2} c_{2}}\left(1-\lambda_{1}\right)}{|A|}+\frac{-v_{g_{1}} \beta_{1} \beta_{2} v_{c_{1} c_{1}} v_{c_{2} c_{2}}}{|A|} \\
& =\frac{-v_{g_{1}} \beta_{1} \beta_{2} v_{c_{1} c_{1}} v_{c_{2} c_{2}}\left(2-\lambda_{1}\right)}{|A|} .
\end{aligned}
$$

Because $\lambda_{1}<2$ and $|A|<0$, it follows from the increasingness and strict concavity of $v$ that both the numerator and denominator on the right-hand side of the last line in (A.36) are negative. Thus, this fraction is positive, which establishes part (i) of the Proposition.

Because $v_{c_{1} g}=0$ and $v_{c_{2} g}=0$, the second row of (4.13) vanishes. Hence, from (A.33),

$$
\frac{\partial c_{1}^{*}}{\partial w_{2}}=\left(1-\lambda_{1}\right) \frac{\partial c_{1}^{*}}{\partial \beta_{1}}+0
$$

Proposition 7 applies because $v_{c_{1} g}$ is nonnegative. Hence, $\partial c_{1}^{*} / \partial \beta_{1}>0$. But $\lambda_{1}>1$, so the expression in (A.37) is negative, which establishes part (ii) of the Proposition.

An analogous argument using the first row of (4.12) shows that

$$
\frac{\partial c_{2}^{*}}{\partial w_{2}}=\left(1-\lambda_{1}\right) 0+\frac{\partial c_{2}^{*}}{\partial \beta_{2}}=\frac{\partial c_{2}^{*}}{\partial \beta_{2}}
$$

The adjusted welfare weights enter symmetrically in the reduced-form optimization problem. Consequently, the comparative statics of $\beta_{1}$ and $\beta_{2}$ are identical once the roles of the two individuals are permuted. Thus, it follows from Proposition 7 that $\partial c_{2}^{*} / \partial \beta_{2}>0$, which establishes part (iii) of the Proposition.

Finally, because increasing $w_{2}$ decreases $\beta_{1}, v_{c_{1}}$ must increase in order to satisfy the first-order condition (3.10). Thus, from (3.12), person one's marginal tax rate must increase, which establishes part (iv) of the Proposition. 


\section{References}

Boadway, R., Cuff, K., Marchand, M., 2000. Optimal income taxation with quasi-linear preferences revisited. Journal of Public Economic Theory 2, 435-460.

Boadway, R., Keen, M., 1993. Public goods, self-selection and optimal income taxation. International Economic Review 34, 463-477.

Boadway, R., Pestieau, P., 2004. Tagging and redistributive taxation, unpublished manuscript, Department of Economics, Queens University and Centre de Recherche en Economie Publique et de la Population, Université de Liège.

Cebreiro-Gómez, A., 2002. Optimal income taxation, production efficiency and mobility of labour, unpublished manuscript, Department of Economics, University of Essex.

Christiansen, V., 1981. Evaluation of public projects under optimum taxation. Review of Economic Studies 48, 447-457.

Diamond, P. A., 1998. Optimal income taxation: An example with a U-shaped pattern of optimal marginal tax rates. American Economic Review 88, 83-95.

Edwards, J., Keen, M., Tuomala, M., 1994. Income tax, commodity taxes and public good provision: A brief guide. FinanzArchiv 51, 472-487.

Guesnerie, R., 1995. A Contribution to the Pure Theory of Taxation. Cambridge University Press, Cambridge.

Guesnerie, R., Seade, J., 1983. Nonlinear pricing in a finite economy. Journal of Public Economics 17, 157-179.

Hamilton, J., Pestieau, P., 2002. Optimal income taxation and the ability distribution: Implications for migration equilibria. International Tax and Public Finance, forthcoming.

Lollivier, S., Rochet, J.-C., 1983. Bunching and second-order conditions: A note on optimal tax theory. Journal of Economic Theory 31, 392-400.

Mirrlees, J. A., 1971. An exploration in the theory of optimum income taxation. Review of Economic Studies 38, 175-208.

Mirrlees, J. A., 1976. Optimal tax theory: A synthesis. Journal of Public Economics 6, 327-358.

Myles, G. D., 1995. Public Economics. Cambridge University Press, Cambridge.

Nava, M., Schroyen, F., Marchand, M., 1996. Optimal fiscal and public expenditure policy in a two-class economy. Journal of Public Economics 61, 119-137.

Pigou, A. C., 1947. A Study in Public Finance, 3rd Edition. Macmillan, London.

Rochet, J.-C., 1987. A necessary and sufficient condition for rationalizability in a quasilinear context. Journal of Mathematical Economics 16, 191-200.

Röell, A. A., 1985. A note on the marginal tax rate in a finite economy. Journal of Public Economics 28, 267-272.

Samuelson, P. A., 1954. The pure theory of public expenditure. Review of Economics and Statistics 36, 387-389.

Spence, A. M., 1974. Market Signaling: Information Transfer in Hiring and Related Screening Processes. Harvard University Press, Cambridge, MA. 
Spivak, M., 1965. Calculus on Manifolds. W. A. Benjamin, Menlo Park, CA.

Statistics Canada, 1998. National Economic and Financial Accounts, Quarterly Estimates 1961-1992. Statistics Canada, Ottawa, Catalogue No. 13-001-SPB.

Stiglitz, J. E., 1983. Self-selection and Pareto efficient taxation. Journal of Public Economics 17, 213-240.

Tuomala, M., 1990. Optimal Income Tax and Redistribution. Clarendon Press, Oxford.

Weymark, J. A., 1986. A reduced-form optimal nonlinear income tax problem. Journal of Public Economics 30, 199-217.

Weymark, J. A., 1987. Comparative static properties of optimal nonlinear income taxes. Econometrica 55, 1165-1185. 